\title{
La sustentabilidad en tiendas de conveniencia de la ciudad industrial en Villahermosa. Tabasco, para el desarrollo de una propuesta
}

\section{Sustainability in convenience stores of the industrial city, in Villahermosa. Tabasco, for the development of a proposal}

\author{
ARIAS-RODRÍGUEZ, Nancy Estela†*, MOREJÓN-SÁNCHEZ, Juana María, ANGELES-GUZMÁN, \\ Casandra y REYES-OSORIO, Yaitla Aitza
}

Tecnológico Nacional de México/ Villahermosa. Km. 3.5 Carretera, Villahermosa - Frontera, Cd Industrial, 86010 Villahermosa, Tab.

ID $1^{\text {er }}$ Autor: Nancy Estela, Arias-Rodríguez / ORC ID: 0000-0002-7297-9153, PubMed Autor ID: ca61c38b5ad13aa82092d2eed967739ce908, CVU CONACYT ID: 921506

ID $1^{\text {er }}$ Coautor: Juana María, Morejón-Sánchez / ORC ID: 0000-0002-9930-181X, PubMed Autor ID: ad978b28511c360eaf67c0997bf9be92e908, CVU CONACYT ID: 362413

ID $2^{\text {do }}$ Coautor: Casandra, Angeles-Guzmán / ORC ID: 0000-0002-2902-6285, CVU CONACYT ID: 914529

ID $3^{\text {er }}$ Coautor: Yaitla Aitza, Reyes-Osorio / ORC ID: 0000-0003-2158-8475

DOI: $10.35429 / J O C S .2019 .18 .6 .23 .27$

Recibido: 09 de Enero, 2019; Aceptado 24 de Marzo, 2019

\section{Resumen}

En la presente investigación se analiza la sustentabilidad de las tiendas de conveniencia de la cd industrial en Villahermosa, Tabasco. Considerando el comportamiento de la variable de investigación (sustentabilidad) en los diferentes contextos internacional, nacional y local. Así como los fundamentos teóricos en los cuales se sustenta esta variable. Se realiza un diagnóstico sobre la sustentabilidad de las tiendas de conveniencia, generando un escenario integral, para así diseñar una propuesta. La herramienta que se utilizó fue la escala de Likert de donde se diseñó un instrumento para determinar de qué manera influyen cada una de las variables cultural, económica, social, política, tecnológica y ambiental (variables independientes o del contexto) sobre la variable de investigación (sustentabilidad de tiendas de conveniencia). Estas variables del contexto están en constantes cambios y movimientos e influyen de manera directa sobre la sustentabilidad de las tiendas de conveniencia ya sea de manera positiva o negativa. El instrumento se aplicó en las tiendas de conveniencia de la cd industrial de Villahermosa, Tabasco, con el fin de obtener resultados determinantes para la sustentabilidad de las tiendas de autoservicio, se utilizó la herramienta de Excel versión 2010, en el cual se obtuvieron tablas y gráficas, simplificando el contenido de los datos dando resultados específicos y necesarios para el desarrollo de la propuesta. De los resultados obtenidos se diseña un modelo para mejorar la sustentabilidad de las tiendas de conveniencia de la cd industrial en Villahermosa Tabasco con lo que se logrará un crecimiento que estará vinculado fuertemente a la responsabilidad social para brindar atención a los trabajadores que forman parte de ellas y a la disminución del impacto en el entorno que las rodea. Asegurando la competitividad, rentabilidad y permanencia de las tiendas de conveniencia.

Sustentabilidad, Crecimiento, Social

\begin{abstract}
This research analyzes the sustainability of the convenience stores of the industrial cd in Villahermosa, Tabasco. Considering the behavior of the research variable (sustainability) in different international, national and local contexts. As well as the theoretical foundations on which this variable is based. A diagnosis is made on the sustainability of convenience stores, generating a comprehensive scenario, in order to design a proposal. The tool that was used was the Likert scale from which an instrument was designed to determine how each of the cultural, economic, social, political, technological and environmental variables (independent or context variables) influence the research variable (sustainability of convenience stores). These context variables are in constant changes and movements and directly influence the convenience stores either positively or negatively. The instrument was applied in the convenience stores of the industrial cd of Villahermosa, Tabasco, in order to obtain decisive results for the sustainability of the self-service stores, the Excel tool version 2010 was used, in which tables were obtained and graphics, simplifying the content of the data giving specific and necessary results for the development of the proposal. From the results obtained, a model is designed to improve the sustainability of the convenience stores of the industrial cd in Villahermosa Tabasco, which will achieve a growth that will be strongly linked to social responsibility to provide attention to the workers who are part of them and the reduction of the impact on the surrounding environment. Ensuring the competitiveness profitability and permanence of convenience stores.
\end{abstract}

Citación: ARIAS-RODRÍGUEZ, Nancy Estela, MOREJÓN-SÁNCHEZ, Juana María, ANGELES-GUZMÁN, Casandra y REYES-OSORIO, Yaitla Aitza. La sustentabilidad en tiendas de conveniencia de la ciudad industrial en Villahermosa. Tabasco, para el desarrollo de una propuesta. Revista de Sociología Contemporánea. 2019. 6-18: 23-27.

\footnotetext{
* Correspondencia del Autor (correo electrónico: nancye894@ hotmail.com)

$\dagger$ Investigador contribuyendo como primer autor.
} 


\section{Introducción}

Hoy en día la sustentabilidad es de gran importancia ya que toma en cuenta múltiples aspectos que van desde la satisfacción y bienestar de sus empleados, la calidad de sus productos, el origen de sus insumos, hasta el impacto ambiental de sus actividades, sin dejar de lado el efecto que causan sus productos y desechos, es decir, el impacto social, político y económico que produce su actividad y su compromiso con el desarrollo social y económico de un país.

Los clientes son cada vez más exigentes y los grupos necesitan una sustentabilidad en todos sus contextos para una buena satisfacción y así lograr la preferencia y mantenerse en el Mercado.

En la actualidad, la sustentabilidad es un tema muy popular en los diálogos y acuerdos entre países y gobiernos; sin embargo, pocos conocen con claridad cuál es su significado e importancia.

La sustentabilidad es en realidad "un proceso" que tiene por objetivo encontrar el equilibrio entre el medio ambiente y el uso de los recursos naturales.

La humanidad en su paso por el planeta ha degradado los recursos naturales de tal forma que actualmente es necesario procurar y planear para garantizar su existencia en las generaciones futuras.

En la presente investigación se analizan las variables del contexto social, económica, ambiental, tecnológica, cultural y política, y sus efectos sobre la variable de investigación (sustentabilidad), para la creación de un modelo de desarrollo sustentable que permita dar respuesta a los contextos y puedan mantenerse en un nivel competitivo que ayude con su crecimiento para estar a la vanguardia de lo que la sociedad exige y así poder ser productivos y competitivos en nuestra sociedad.

Actualmente en las tiendas de conveniencia de la cd. Industrial de Villahermosa, Tabasco se ha observado que la sustentabilidad no corresponde al grado que la Sociedad demanda.
La sociedad demanda un servicio de calidad donde se cumplan sus expectativas en todos los ámbitos (económico, social, tecnológico, cultural, ambiental y político), añadido a la alta competencia y a la apertura de opiniones en las redes sociales, es por lo cual se hace este análisis de cómo afectan las variables del contexto (económica, social, tecnológica, cultural, ambiental y política) en la sustentabilidad, donde se toma una actitud socialmente responsable para dejarle a la siguiente generación un mundo estable, utilizando las diferentes estrategias para emplear, salvaguardar y mantener los recursos humanos y materiales de manera óptima, con el objetivo de crear un equilibrio responsable y beneficioso, sostenible a largo plazo, mediante la recuperación y el reciclaje con una administración eficiente y racional de los recursos naturales, de manera tal que sea posible mejorar el bienestar de la población que afectan la sustentabilidad de la empresa.

La hipótesis formulada en esta investigación, son las variables económica, social, tecnológica, cultural, ambiental y política que influyen en la sustentabilidad de las tiendas de conveniencia de la cd. Industrial de Villahermosa, Tabasco.

\section{Descripción del Método}

El levantamiento de la información se hace mediante un instrumento (cuestionario, Tabla 1), utilizando la técnica de Delphi el cual se desarrolló para obtener el diagnóstico de la situación actual, con el fin de obtener un consenso de la opinión de expertos para identificar los puntos claves para realizar una propuesta de mejora. Uno de los principales objetivos de la investigación es obtener información mediante el instrumento y determinar si las tiendas de conveniencia son o no son sustentables. El instrumento (encuesta tabla 1), se aplicó en las tiendas de conveniencia de cd. Industrial de Villahermosa, Tabasco, Con el fin de obtener resultados determinantes, se utilizó la herramienta Excel versión 2010, mediante el cual se obtuvieron tablas, graficas; simplificando el contenido de los datos, dando resultados más específicos y necesarios para el desarrollo de la propuesta. 
ENCUESTA

NOMBRE DE LA TIENDA DE CONVENIENCIA:

INSTRUCCIONES:

Marque con una $\mathrm{X}$ el criterio que usted considere conveniente de acuerdo a los diferentes criterios.

5) Muy de acuerdo

4) De acuerdo

3) $\mathrm{Ni}$ de acuerdo, ni desacuerdo

2)En desacuerdo

1) Muy en desacuerdo

\begin{tabular}{|l|l|l|l|l|l|}
\hline & 5 & 4 & 3 & 2 & 1 \\
\hline
\end{tabular}

Variable tecnológica

1.- Es importante la aplicación de la tecnología dentro de las tiendas de conveniencia

2.- La creación de una página facilita la búsqueda de los productos

3.- El personal cuenta con el conocimiento para la utilización de herramientas tecnológicas

Variable ambiental

1.- Le parece bien utilizar bolsas de papel para dar ejemplo de mejora a medio ambiente

2.- La ambientación física de la tienda es la adecuada

3.- Considera que el no colocar recipientes adecuados para tirar basura, contamina el medio ambiente de la tienda

4.- Considera adecuado el ambiente con sus trabajadores

Variable cultural

1.- Sus valores y principios le permiten comprar en la tienda de conveniencia

2.- Se encuentra muy a gusto con la valoración de su trabajo que realiza

3.- La creencia cultural de la sociedad y sus valores influyen en el servicio que prestan la tienda de conveniencia

4.- Usted como trabajador se siente motivado con el servicio que proporciona la tienda

Variable social

1.- Estaría dispuesto a ser parte de campañas para cambiar la mala cultura y cuidar el medio ambiente

2.- La tienda es importante para el desarrollo de la cd. Industrial

3.-Considera buena la relación que tiene con los consumidores

4.-Cree usted que la remodelación en las tiendas podría darle un mayor impacto en la comunidad de cd. Industrial

\section{Variable económica}

1.- $\quad \mathrm{Se}$ considera sustentable

económicamente a la tienda

2.- Considera que la tienda es de apoyo para la economía del Estado

3.-Considera bien que la sociedad ayude al desarrollo de la tienda en cuanto a dar el redondeo cuando no llega al peso las compras

Variable política

1.- Cumple con los requisitos establecido por la ley nacional de tiendas

2.- Cumple con los estándares de clasificación, distribución y áreas

3.- Está usted de acuerdo que las políticas emitidas por distintos organismos gubernamentales le han sido de beneficio 4.- La creación de nuevas reformas políticas tienen efecto en las tiendas de conveniencia

Tabla 1 Encuesta aplicada a las tiendas de conveniencia de la Cd. Industrial de Villahermosa, Tabasco

Fuente: percepción del autor 2019

\section{Análisis de la información}

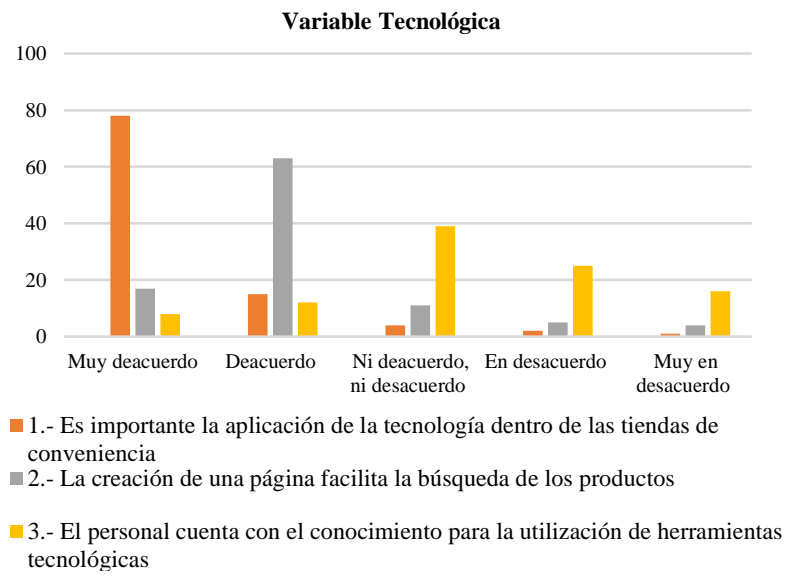

Gráfico 1 Variable Tecnológica

Fuente: percepción del autor 2019

Variable tecnológica, el $78 \%$ de las tiendas de conveniencia considera muy importante el uso y la aplicación de la tecnología dentro de las instalaciones, sin embargo, un 63\% considera que la creación de una página les facilitaría la búsqueda de los productos, aunque un $61 \%$ considera que el personal no cuenta con el conocimiento de usar las herramientas tecnológicas (gráfico 1).

Variable Ambiental

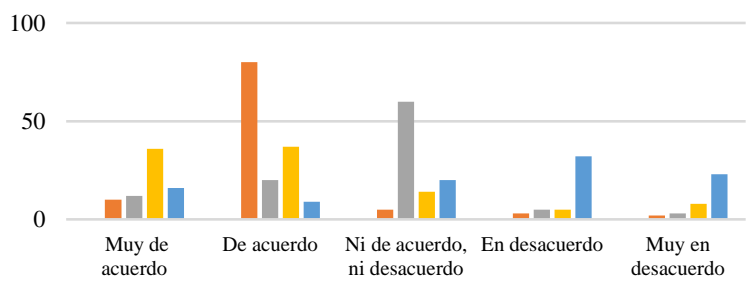

1.- Le parece bien utilizar bolsas de papel para dar ejemplo de mejora a medio ambiente

2.- La ambientación física de la tienda es la adecuada

$\square$ 3.- Considera que el no colocar recipientes adecuados para tirar basura,

contamina el medio ambiente de la tienda
4.- Considera adecuado el ambiente con sus trabajadores

Gráfico 2 Variable Ambiental

Fuente: percepción del autor 2019

En la variable Ambiental un $80 \%$ considera que se deberían sustituir las bolsas de plástico por bolsas de papel y un 50\% considera que el no poner contenedores adecuados perjudica el ambiente ya que los desechos son dejados en lugares inadecuados y se encuentran muy a menudo con problemas de desechos (gráfico 2). 


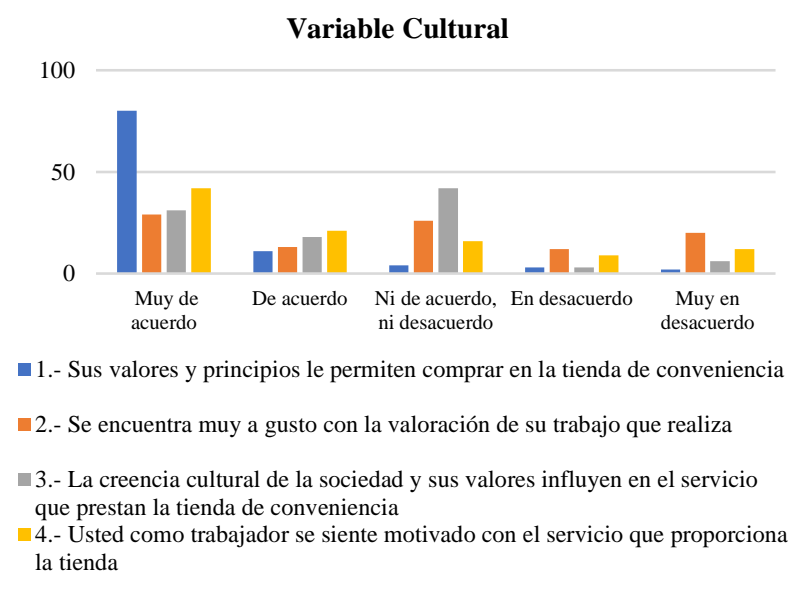

Gráfica 3 Variable Cultural

Fuente: percepción del autor 2019

En la variable cultural, un $80 \%$ manifiesta que los valores y principios que se tienen como persona son de suma importancia para una convivencia agradable con los empleados por lo que un $42 \%$ se siente motivado con el servicio que presta la tienda (gráfico 3).

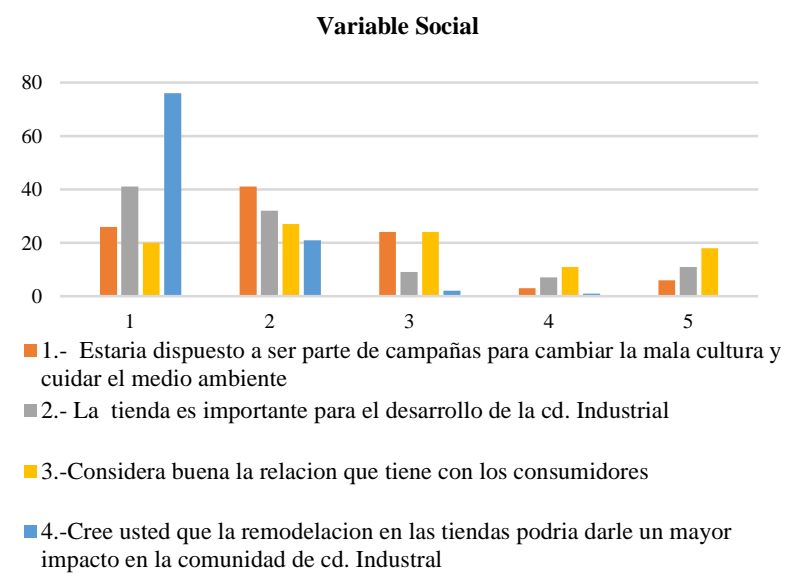

Gráfica 4 Variable Social

Fuente: percepción del autor 2019

En la variable social, el $76 \%$ consideran importante que las tiendas requieren remodelación para tener un mayor impacto en la sociedad ya que un $40 \%$ la considera importante para el desarrollo de la ciudad (gráfico 4).

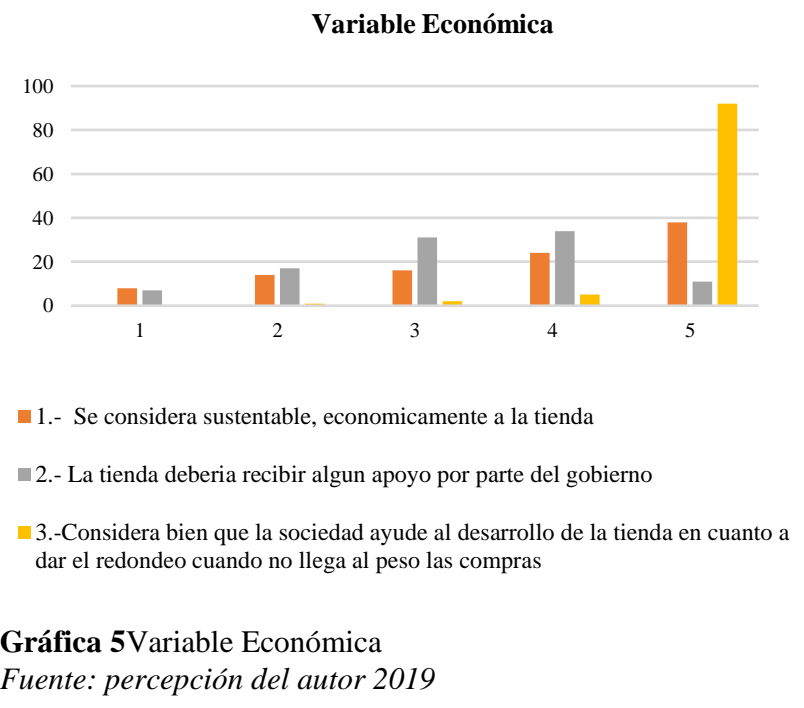

En la variable económica, un $90 \%$ no quiere dar apoyo económico para su crecimiento, ya que considera que la tienda tiene fondos suficientes para invertir sin ayuda de sus clientes. Un 20\% de las tiendas no se consideran adecuadamente sustentables económicamente (gráfico 5).

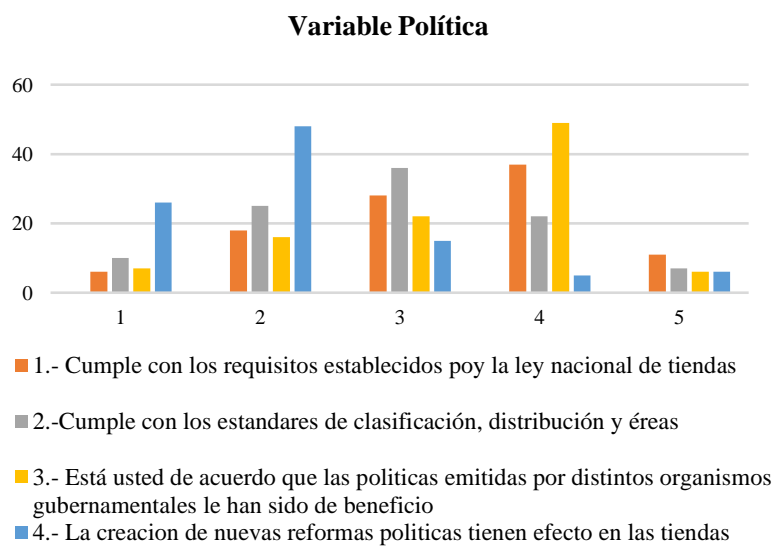

Gráfica 6 Variable Política

Fuente: percepción del autor 2019

Y la variable política, un $36 \%$ cumple con los requisitos por la ley nacional, y también con el cumplimiento de clasificación, distribución y áreas, así como el 30\% está de acuerdo con las políticas emitidas por parte del gobierno, como también un 35\% de acuerdo en la creación de reformas tienen efecto en las tiendas (gráfico 6). 


\section{Resultados}

Todas las variables independientes han impactado en la variable de investigación. Pero las variables que más impactaron, fueron la tecnológica, Ambiental y económica; por lo que se procederá a diseñar una propuesta a través del diseño de un modelo que puede considerarse para 1 solución de la problemática planteada al principio del proyecto. El modelo (Figura 1). Se diseñó en base al impacto de las variables de contexto sobre la sustentabilidad de las tiendas de conveniencia.

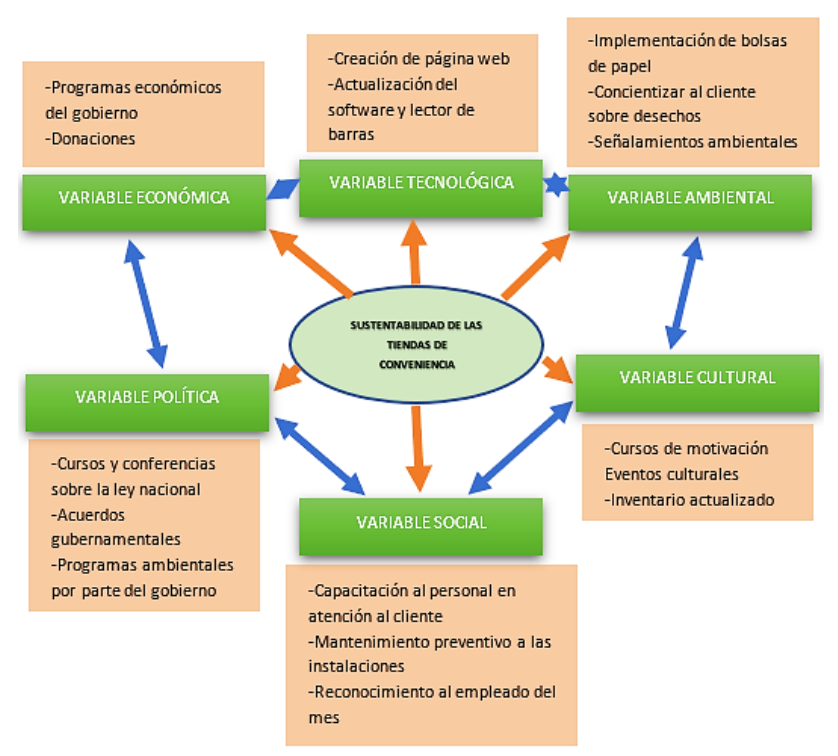

Figura 1 Modelo para mejorar la sustentabilidad en las tiendas de conveniencia de la cd. Industrial de Villahermosa, Tabasco

Fuente: percepción del autor 2019

\section{Agradecimiento}

Gracias a Dios, al Tecnológico Nacional de México/ Villahermosa, y a nuestros seres amados.

\section{Conclusiones}

La realización de la investigación a todas las tiendas de conveniencia que cuenta la ciudad industrial en Villahermosa Tabasco, permitió conocer un panorama integral de las tiendas, y a partir de los resultados obtenidos se mostró que las variables económica, ambiental y tecnológica tienen un mayor impacto sobre la sustentabilidad de las tiendas. Las variables del contexto están en constantes cambios y movimientos e influyen de manera directa a las tiendas de conveniencia ya sea de manera positiva o negativa, con este modelo de mejora para la sustentabilidad se podrá lograr la permanencia en el mercado teniendo clientes totalmente satisfechos.

\section{Referencias}

Albert G. H. Luis Zuñiga L. (2012). El Desarrollo Sustentable y las organizaciones. México: Sauyed.

Calvente, A. M. (2007). El concepto moderno de sustentabilidad. México.

Coutiño, R. D. (2012). Desarrollo sustentable: una oportunidad para la vida. D.F.: McGraw Hill.

Juárez, G. D. (2011). Desarrollo sustentable en el contexto actual. México: McGill.

Juliet Beth Zimerman, James R. Mihelci. (2015). ingeniería ambiental. México: Alfaomega.

Méndez, I. A. (2014). definición integral de una sociedad sustentable. Un mejor mundo, 16 y 17.

Morejón Sánchez Juana María (2018) Sustentabilidad de las bibliotecas públicas en la ciudad de Villahermosa, Tabasco, para el desarrollo de una propuesta. ISSN 1946-5351 Vol. 10, No. 2, 2018

Suárez, M. V. (2014). Desarrollo Sustentable. México: Grupo Editorial Patria.

Torres, E. G. (2011). Desarrollo sostenible. México: Alfaomega 